\title{
The brain and mind as a system.
}

\author{
Thomas Saaty* \\ Center of the Philosophy of Science, University of Pittsburgh, USA
}

\begin{abstract}
The aim of this paper is to provide a sketch for how to look at the nervous system and its byproducts of mind and feelings and how they function in a general philosophical way as a system with consciousness and with purpose. We identify a diversity of situations and challenges of performance. Our goal in doing it is to give to the reader the tools needed to be able to combine the more technical mathematical study of the nervous system with its major functions and purposes.
\end{abstract}

Keywords: Nervous system, Mind, Brain.

Accepted on June 03, 2017

\section{Introduction}

We find it convenient and aesthetically satisfying to think of systems in terms of four major attributes in which flow and function are part of the structure, as illustrated in Figure 1. These are: purpose, function, flow and structure. Selfreproduction is part of function and is not a characteristic of all systems. The brain that has the creation of the mind and feelings as a purpose. One proceeds from the most general purpose to the functions of the parts; the flows necessary to perform the functions and the structure (such as the brain itself) which constrains and directs the flows of neurotransmitters and electric firings of neurons that mostly use synapses to communicate with each other. This framework also makes it possible to differentiate and categorize a variety of systems. We note that the entire universe is one gigantic system. From a practical standpoint, we do not deal with the entire universe every time we have a problem. Thus, a particular system is a subsystem of the universe that in the broadest sense is its environment.

There is a distinction to make between purposive and purposeful systems. Purposive systems behave as programmed robots. They are tools used to fulfill needs perceived by their designers. Refrigerators serve the purpose of keeping food fresh; homes serve the purpose of protection from the environment; automobiles and airplanes serve the

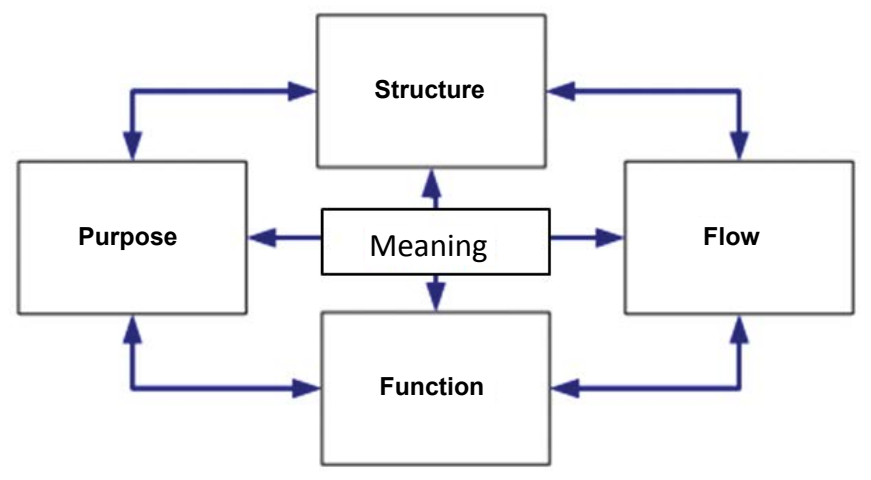

Figure 1. The brain as a system. purpose of transportation. Purposeful systems are inventive; examples are people and animals, universities and hospitals. Their purposes arise from their ability to perceive needs to pursue alternative actions to satisfy those needs. They are also dynamic: their purposes and their style of selecting them change over time.

Both kinds of systems are open in that they exchange material or energy with their environment yet the inventive variety are distinguished by its ability to consider responses to environmental constraints. Systems that serve a purpose merely react to the environment with programmed outputs.

We classify preferred outcomes in terms of the amount of time required to bring them about. Objectives are preferred outcomes attainable within a specified and relatively short period. Goals are attainable in longer time and require the prior satisfaction of one or more objectives; ideals are states of a system never attained but merely approached with satisfaction of some objectives. Just as goals are clusters of objectives, ideals are clusters of goals.

It may be worth mentioning that purpose itself need not be deterministic. It can develop over time and is not necessarily unique. An interesting interpretation of this idea is due to $\mathrm{J}$. Monod, who won a Nobel Prize in "Physiology or Medicine" in 1965 . His thesis is that life is all a combinatorial accident based on chemistry and physics.

Purpose is a behavioral concept that is subjective and hard to define. Many people whose thinking is based on principles drawn from the natural sciences and engineering prefer to confine the definition of purpose to structure and flow and their interactions. Matter and motion are as intrinsic purposes. However, in social systems, human behavior is a powerful force that we do not understand adequately but that nonetheless appears to shape our approach to reality. It is difficult for us to understand systems, particularly those involving people, without reference to a human purpose. There is uncertainty in our attempt to define a system by looking at things we identify as systems. We must often modify our 
definitions as we become more and more specific. When one attempts to define a human as a system, causal explanations of the ultimate purpose of why people are designed the way they are fail to explain what their ultimate purpose is and how this design really contributes to that purpose. One often encounters circular arguments between human design and human purpose.

\section{Structures}

Like many words, structure refers to a wide variety of concrete objects and abstract ideas, yet it is generally possible to understand its meaning from the context in which it is used. We can usually form some preliminary conclusions when a neighbor tells us that his new home is structurally defective. The foundation is shallow or the walls may be warped. When someone tells us that his life is structured, we can immediately surmise that he is referring to a high degree of repetition of tasks and sequencing of daily activities. In the vocabulary of systems theory, structure has specific conceptual meaning. First, we must distinguish between the notions of form, aggregate and structure.

Form is the external overall appearance of an object without regard to its substance but with due regard to the organization of its parts. Two objects such as a ball of string and a ball of wax may have a different content but the same form. A form is a geometric concept that refers to an assemblage of things or ideas. An aggregate is the conjunction or collection of particulars into a whole mass or sum without regard to form or order, like things in a hiker's backpack.

A structure is a set of parts or forms that relate together in a specific order (holism) to perform a function. The structure has the capacity to endure over time through cohesive adjustment to maintain the parts and their relation in nearly the same order by responding to the pressures of the environment.

Structures are linkages of substructures or components, and these in turn are linkages of elements. All linkages may allow some kind of flow. The structural composition and arrangement of a plant is such that water, nutrients, and solar energy pass through its structure, thereby sustaining life. Any structural damage may impede the plant's function, leading to structural decay. We observe that the structure of a system is adapted to its functional transformations; and it works to facilitate these transformations. This relationship involves circularity and mutual dependence.

In organizations and in the broader social structure, there are cohesive forces that maintain the form of the structure. Externally generated forces are those whose origins are in the social structure itself. They include norms of behavior, mores, codes of ethics, and so on. They pattern our individual behavior and form linkages between ourselves and other likeminded persons or groups. Internally generated forces are those unique to the individual. They include personal needs such as physical sustenance, recreation, companionship, selffulfillment, and the like. There are the needs that prompt each of us to form some linkages with those around us; these needs are very strong and unique to the personality.
Characterizing the arrangement and linkages between elements in social structures or organizations presents many problems, since each element is itself a purposeful system. As individuals and as groups we have at least a limited amount of control over our placement within the social structure and we determine, in part, the extent to which we interact with other components. As systems, we act in self-determined ways, making the notion of a social structure more elusive than that of a mechanical or biological structure. Structure in the social realm refers to an abstraction-something that can be perceived and whose boundaries may be conceptualized; yet it cannot be dissected in the manner of mechanical or biological structures.

For practical reasons we must simplify the representation of structure in order to identify its elements and their interconnections. One of the simplest models for such a representation is a network. The network concept serves to describe the actual "structural" connections of the elements of the system, as in transportation or it may be an abstract "functional" representation of interactions between the components, as in a hierarchical representation of the various levels of regulation and control of an organization. Social network analysis has recently become a popular tool for studying the spread or diffusion of ideas in society. For example, several researchers have employed network analysis to explain and predict the diffusion of innovations among state and local government officials. In this context, it is illustrative to note that flow of information within social networks involves the diffusion "infrastructure" characterized by formal linkages between producers, suppliers, and potential users of innovations.

Organizational structures change with the purposes of their designers, managers, and components. As new needs arise, new functions identified, and new flows designed to serve functions. They also change through growth and evolution as they respond to perturbations from the environment, assuming new structural arrangements that make them less vulnerable to damage in the future. The enactment of the myriad social welfare laws following the Great Depression and of civil rights legislation, followed the racial disturbances of the 1960s are examples of structural evolution. Often, restructuring is a temporary "tactical" response to conditions in the environment that gives the system time to formulate a holistic or "strategic" response. For example, the contingency theory of organizational development states that firms are more likely to decentralize their internal structural arrangements in response to rapidly changing environmental conditions, such as intense competition, introduction of new products, changing economic conditions, and the like. Conversely, under relatively static environmental conditions the organization is more likely to assume centralized and rigidly ordered hierarchical arrangement. Restructuring; always brought about to facilitate new flows that perform functions in pus of new goals and objectives. Thus organizes generally are thought to be self-organizing systems.

\section{Flows}

All functions are a result of flows of energy, materials, people 
or information. A system may have several simultaneous flows. Here are some examples of flows:

- Flow of water in pipes or electricity in wires or neurons

- Flow as transport and storage of matter

- Flow as transduction and radiation of energy

- Flow as transformation of information

- Flow as conversion from one state to another

- Flow as energy cost of triggering energy release

Flow is movement of material and energy. A structure is essentially a set of constraints on the flows in space and in time. It channels them along designated routes and subjects them to various transformations with time lag, sometimes allowing for regulation and feedback. The structure itself may change in time and may be subject to transformation by the flow, as is the case with wear out due to continuous flow. The structure (which is a system within the system) may also change due to growth, evolution, or decay; thus structure, in turn, affects the efficiency of the flow. Flows essential to stabilize or to keep the primary structure together (such as the flow of blood to the tissue) are called sustaining flows. Other flows that are the result of structural performance may be waste flows or product flows. Fluid movement provides a simple example of how flow through pipes is constrained by the primary structure. Some of the more complex flows such as diffusion, radiation or communication are also constrained by the structures through which they pass, albeit in more subtle ways than fluid flows. For example, the flow of communication in hierarchical organizations fall in certain "channels" whose boundaries are not defined in discrete physical terms but whose influence is, nevertheless, very real. The tradition of the organization, norms of behavior, unwritten rules of interaction and simple common sense need to know "who talks to whom". In this way, the organizational structure imposes constraints on the flow of communication and facilitates the efficient operation of the system.

Several levels of flow exist in any structure. For example, there are flows in and out of red blood cells, that are themselves part of blood flow. There are flows at the level of the organic molecules that build these cells. There are flows among the atoms that in turn are part of the organic molecules. The human body contains many flows that interact with one another. None of these flows may appear meaningful by itself to the human mind. Together, however, the flows maintain life.

The reasons why transformation and change, realized through flow, are so basic to understanding is that consciousness by definition requires change from identity to diversity so that it can recognize things [1]. To be conscious is to introspect and internalize the observation of change. The idea of transformation is associated with combining things in special ways to produce new entities (that is, the law of composition). To change is to seek variety. Change is the most basic attribute of all existence. For a system to fulfill its function, it must seek change from instant to instant even if the process repeats.
Flows, like the structures that constrain them, do not remain static over time. As the structural composition of a system changes, the character of the flow changes as well. Many management innovations such as Zero-Based Budgeting require structural reorganization that dramatically alters intraorganizational flows of communication and resources. Flows can also change without preceding or lagging behind changes in structure. For example, plants and certain animals enter dormant periods during which the structural and functional arrangement of components remain the same, yet the flows of food and energy sources are altered; that is, the metabolic process slows down. Physical flows require a driving force to propel material or energy through the structure. A pump or gravity pushes fluid through pipes and transmitters send radio and television signals through the air. There is a correspondence between the power of the driving force and the rate and direction or distance of flow.

In social systems, we find that we cannot precisely isolate cause/effect relationships between driving forces and flows. Social forces such as prevailing economic conditions in a certain region may partially determine flows of people into and out of that area; but it is not possible to measure precisely the relationship between forces and flows. The electrical system of our body is composed of 3 parts: (1) The brain, the central nervous system, (2) Spinal cord which is inside of the vertebral column and serves as an electrical cable. (3) The peripheral nerves - the electrical wires - that connect the spinal cord to all parts of our body.

\section{Functions}

Functions are transformations of flows into action. These actions are events that realize purpose. It is useful to think of the function of a component as an aggregate of its states in space and time. For example, the function of a wheel is the collection of all its positions as it rotates; when the wheel is not turning, we believe that it is not performing its proper function.

Functions may build upon one another in a sequential fashion, as in an assembly line. At each station, tasks add a new feature or dimension to the product. The process continues until the product emerges in its final form. In this example, one might take a broader perspective by interpreting the entire assembly line as a single functional component of the larger system in which it is part, a manufacturing plant. Other functions such as product design, testing, marketing, and research and development made in relative isolation; yet they complement each other in the realization of purpose of the entire operation.

Interaction between the functions of two or more components often has an unanticipated or emergent, quality that not found in any single component. For example, it is difficult to understand the purpose of an isolated automobile part; but its purpose becomes clear when we see the part interact with other components as the car works.

The same function serves in several different ways. Systems can be classified by the freedom or latitude displayed in the performance of functions. In mechanical or robotic systems, the designer programs functional transformations into the 
system. The purpose of the functions is to maintain the system in a steady state of operation. The set of functional transformations performed by any mechanical system is finite and the system has no choice in deciding that.

In biological systems, functional transformations are determined by the genetic code; yet they are also modified by changes in the conditions of the environment. A biological system may not choose its purpose or objective, but it may have a choice of functions to achieve the programmed objective. For instance, organs in the human body secrete a wide variety of antibodies to combat viruses. If one antibody fails, the organ may send another type to accomplish the objective. Biological functions can change in the face of different environmental influences.

Man as a purposeful system has the ability to perform the same function in different ways. Naturally, the number of ways of performing a function is constrained by the purposes serves. However, human systems generally have a choice from among several strategic or tactical functions in most of their pursuits.

\section{Human Purpose}

Man is distinguished from other systems by an ability to perceive and differentiate among alternative courses of action and to design and control his own actions by a conscious effort. He establishes priorities and makes a choice through preference, necessity, caprice or whatever other forces. The activities of the brain do not proceed in a random manner. They exist through programs that give rise to homeostasis and the continuation of life. These aims stem from the actions of relatively small central parts, still little understood. The reticular system serves to activate both what comes in and what goes out, keeping the whole organization going. The hypothalamus is located in front of this system, lying at the base of the brain. Its cells monitor the condition of the blood and tissue compelling instructions if for instance there is too little sugar or water or too much carbon dioxide. These areas therefore set the aims of the whole individual.

Daily we deal with mostly routine matters, but the accumulated response to these smaller choices over time still has huge impacts. These issues underlie a series of not so routine choices: where to go to school and what to study; whom to marry; when to retire; and sometimes even when to die. These choices, when followed by actions, give rise to new situations or states of existence. The cycle continues as new alternatives arise because of previous choices.

Groups of people form organizations and make choices to satisfy common purposes. Examples are what is made and what to charge for it; what taxes to levy and what streets to pave; what weapons to build and how to fight a battle. In all decisions, the choices made today shape the alternatives of tomorrow.

While the notions of choice and purpose are taken for granted, they are crucial to understand a particular system. We study systems to identify points of intervention where functions, flows, or structure can be modified to satisfy purpose. We also examine the purposes themselves to see if they are feasible or desirable in light of the existing circumstances.

The purpose of a system designed by people is, on the first level, given to it by the designer. Later the users of the system adapt it to their own purposes. An "ultimate" objective or purpose may or may not be perceived, even by the designer, as in the case of the use of nuclear energy. In this sense, a system should always be considered in the context of its users rather than of its designers -since they imbue it with purpose.

When designers and user's basic purposes and viewpoints differ, their descriptions of a system may also differ, particularly when it relates to political and social impacts. This difference could give rise to conflict if actions are needed by one group opposed by another group. A system can have more than one purpose depending on the perspective of the observer. For example, research has demonstrated that some people regard the criminal justice system as primarily a punitive system because it imposes sanctions on violations of the law, while others see it as a means to focus on crime prevention by acting before violations occur. Still others perceive the criminal justice system as a vehicle for delivering many types of social services and assistance to citizens. Clearly, differing perceptions of the purpose of a system can lead to vastly different assessments or measures of the system's performance. Investigators in the field of evaluation research, who attempt to assess the performance of various types of systems, are discovering that many standard evaluation techniques fail to account for multiple and conflicting perceptions of the purpose of a system. It is, therefore, impossible to obtain a complete evaluation of a system's performance in the absence of a synthesis of conflicting viewpoints.

The individual himself may not view the system the same way at all times. His view of it may depend on how it interacts with other systems he also uses. For example, a biologist may sometimes view a virus as a plant, sometimes as an animaldepending on how it behaves in its environment.

An existing system may be thought of by various people (or even by the same individual) to have different purposes. For example, a car engine maybe regarded by an aborigine as a source of heat for cooking while it is regarded by a member of a technological society as a means to transmit energy to rotate wheels. Conversely, a given purpose can be carried out by different systems. For example, one can travel by car, air or horse.

Because purpose is essential to the definition and evaluation of a system and because it is relative to the point of view of the people involved as a part of the system, we must include in our definition the variety of perspectives and possible differences among them, which can lead to conflict. Thus with every system there is a potential for conflict over what its purposes may be and what functions it performs. We refer to the different perspectives of a system as the potential conflict environment. A basic problem in systems theory and particularly in social systems is how to obtain agreement on what the purpose is as a first step to the resolution of conflicts. 
Organizations as systems should not regarded in isolation of their participants. When people are components of systems, their behavior either facilitates or inhibits the fulfillment of purpose. The human element of purposeful systems makes them less predictable and less manageable than mechanical systems.

It follows that for social systems to achieve their purposes people must cooperate with an implicit objective of making the system function better. We need to believe that our contribution to the system is important. It is also better to feel stimulated and creative at the tasks we perform. The quality of our working environment, the intellectual stimulation of our work and our personal investment in the output of a product all contribute to our commitment to the purposes of the system. To create a cooperative atmosphere in a system, a manager must display strong leadership and have an ability to motivate people without manipulating them. Resolving conflicts in a way that keeps the "losers" committed to the organization is a task that requires specialized competence in negotiation strategies, arbitration or organization development.

\section{Purpose and the Environment}

The purposes of a system are linked to the environment in which the system is embedded. We normally consider the environment of a system to be those factors external to the system that influence its behavior. Therefore, we need to study the environment to understand the compatibility of the purposes of the system with the functioning of the environment. A system may appear to be imperfect as far as its perceived objectives are concerned, yet it may be in harmony with the environment. A person who is lame, for example, may still be able to function well. Lack of appreciation of this connection between the individual and the environment can cause us to design systems that are in conflict with their environment. A system with an apparently clear and desirable purpose that relates poorly to the environment would not serve the purpose of its designer. In our time, we have seen beautiful apartment complexes decay quickly because they were not well suited for either their users or the people who lived near them, or their environment. A system relates to the environment through the following criteria:

Purpose is the system compatible with the environment as we know it? If not, one should modify the purpose, abandon or discard the system, or reshape and adjust the environment.

Design involves the assembly of components so that they interact in harmony both among themselves and with the environment to achieve the purpose. The purpose is modified to conform to what it is possible to realize and the cycle repeats.

Evaluation is the system itself actually compatible with the environment? Does it accomplish its purposes efficiently? Does the system in operation suggest additional objectives to us that can be achieved with slight modifications?

A problem which occurs in a given system can be regarded either as improper functioning in the system itself or as a flaw in its interactions with the environment. The interface of the system with the environment is separate from either the system or the environment. Solving one problem can lead to disturbing other factors in the system or in its interface with the environment. Furthermore, solving a problem can disturb a system, even after a considerable lapse of time. For example, achieving the racial integration of schools through mandatory busing produced very noticeable impacts on the public school system more than a decade later. Declining enrollments in public schools has contributed to a cycle of decline in the quality of public education. Moreover, critics claim that mandatory busing may, in fact, intensify racial segregation since minorities cannot afford the private school alternative.

\section{Constraints on the Realization of Purpose}

Despite the best efforts of highly trained analysts and problem solvers, social systems rarely realize originally intended purposes. Here are some notable barriers to the fulfillment of these purposes.

\section{Internal constraints}

Perceptions: Our separate views of the world shape our perceptions of the environment and may enhance or hinder our ability to identify and distinguish among alternative needs and purposes and the means to achieve them.

Unique problems: Often needs, when they occur, are examples and there is no predetermined set of ordered procedures to satisfy them. Each need, or collection of needs, is dealt with anew.

Conflict and power: Perceived needs produce conflict in a system. Decision makers cannot agree on the definition of the problem or else disagree on the purpose itself. Often, after compromising, one finds that the resulting system no longer has its original purpose to which there may have been considerable commitment of energy and principle and may even be simultaneously pursuing multiple and conflicting purposes.

Inertia: Major human decisions sometimes are attributable to uncontrolled sociological forces rather than to individuals. Individuals ossify in bureaucracies and take on lives of their own in the process becoming less sensitive and adaptive.

\section{Environmental constraints}

Dynamics and planning: This constraint mainly has to do with our frustration to predict and plan the future. A large number of factors, their interdependence, and continuous change elude the identification of an "optimal" solution, if indeed there is one. It is not clear what one can achieve in such a "mess."

Turbulence: Complex networks of inter-system relationships, combined with the dynamics of the environment, may create "turbulence" inhibits the ability of any single system to survive and grow based on its own adaptive capabilities.

Delayed feedback: Feedback on the performance of a system or on the appropriateness of its purposes may not 
take place immediately. Since many events may occur in the intervening period, when feedback finally arrives, the results of feedback can be misinterpreted or overlooked and may seem to be of little use in adjusting the behavior of the system. Nevertheless, one need is a way to accommodate feedback to make it possible for adjustments to the system.

These constraints on purposeful choice need to be added to the list of obvious ones, such as limited technical, financial, or human resources. We are coming to the realization that optimization is an ideal. Despite the availability of exotic techniques of optimization, it is not necessarily best in an overall systemic sense to always attempt optimization instead of simply satisficing (In real life, complexity and quick changes in factors and constraints leave little time for the kind of precision that may be aesthetically satisfying, like finding the optimum, but is of no practical consequence. The fact that factors may change or have delayed impact makes it clear why it is often not as critical for us to optimize as it is to be satisfied at some reasonable level. Nature seems to have opted for satisfaction and only occasionally do we have the opportunity to indulge in deliberate optimization consciously.

\section{Values and Purpose}

The traditional argument that science offers only factual rather than ethical or evaluative statements is nowadays considered not very useful in systems thinking - particularly in discussions of purposeful systems. Purposeful systems are not value free, but value laden. They are selective systems that choose from available alternatives based on normative statements such as "should" and "ought." They seek preferred outcomes with significant ethical and evaluative implications. Even apparent objective statements of fact supported by hard data may turn out to rely on normative statements that could be challenged.

Truly value-free research would be devoid of content, for it would not touch our lives in meaningful ways. The presence of values does not mean we have to give up the quest for objectivity. Rather, normative statements of ethics and values can be surfaced for open debate and actually contribute to rational objectivity. They can be treated like statements of fact. It is clear that there is a place for discussions of ethics and values in the conduct of systems research and for methodologies designed to deal with these issues.

\section{Characterization of Open Systems}

Traditionally people have considered three basic categories of systems (1) transition systems, which change from state to state with or without purpose; (2) adaptive control systems which can be made to change from the current state to another desired state by appropriate feedback and information; and (3) learning systems, which can change from state to state thus also changing the purposes of the system (a process that requires imagination adaptability, and originality).

A basic method for characterizing systems in terms of flow is to identify them as open or closed. A system is open when it exchanges matter, energy or information with its environment, importing and exporting building-up and breaking-down its material components.
By definition, the only known perfectly closed system is the universe. Everything is in it, its boundary is empty, and nothing crosses the boundary. The system is closed. A closed system has no interaction with the environment.

The concept of a closed system is only intellectually interesting. To assist understanding we can use approximations of closed systems such as Robinson Crusoe's economic system or the little greenhouse jar. Of course such a system is not closed with respect to the intake of solar energy, for example, but it is closed with respect to transfer of goods and services.

An open system is a system that interacts with its environment. It is relatively easy to accept this definition of an open system, despite the fact that our understanding or knowledge of what defines the boundary of the system and what crosses this boundary is often fuzzy and cannot be readily specified. The environment places constraints on what crosses the boundary. Even an open system is included in some closed system (the universe?). If a system $\mathrm{S}$ were to include a closed system $C, C$ would be impossible to study through the purposes of $\mathrm{S}$ and would have no effect on anything in $S$. Thus there is no reason to consider the inclusion of $\mathrm{C}$ in $\mathrm{S}$. Therefore, we state: (1) Closed systems are not subsystems of any system; (2) Equivalently, all subsystems are open.

A closed system in equilibrium does not need energy for its preservation nor can energy be obtained from it. For example, a closed reservoir contains large amount of (potential) energy; but it cannot drive a motor. The same is true of a chemical system in equilibrium. It is not in a state of chemical rest; rather, reactions are continually going on, regulated by the law of mass action.

We have provided a way to organize our thinking about the brain by considering its four subsystems: structure, flows, functions and purpose. The first two are physical and the second two are in the domain of the non-physical or mental. In doing research one can specialize and focus on any one or on different ones of these subsystems. In this book, we are fundamentally concerned with the mathematics of the functions and purpose arising out of the flows.

"At the outset, let's recall...that emotions are not the disorganizing, irrational, lower-than-human forces that they are often stereotyped to be. Rather, they organize us; they focus our attention and motivation on key goals. Without emotions we cannot perform rational motivated behavior at all. Now still, emotions can be primitive. Recall the reptilian emotions of rage, "fight-or-flight", dating from hundreds of millions of years ago in evolutionary time, without some regulation over them, our lives could be quite difficult indeed...An emotion is a relatively discrete event that typically spans just a few seconds. Certain stimuli tend to trigger happiness, surprise, sadness, anger, fear, and disgust... Classic emotion displays do not last more than 10 s or so..." - Professor Hinshaw Origins of the Human Mind: Emotion Regulation and Mood Disorders."

\section{Alphabetical List of Feelings and Emotions}

Adoration, affection, alarm, agitation, aggravation, 
aggression, agony, alienation, amazement, amusement, anger, angst, anguish, annoyance, anticipation, anxiety, apathy, apprehension, arousal, astonishment, attractiveness, awe. Bitter, bliss, boldness, boredom. Caring, caution, crosspatch, cheerfulness, confidence, contempt, contentment, confusion, compassion, curiosity. Depression, desire, despair, defeatism, delight, dejection, disappointment, displeasure, dislike, distress, dismay, disgust, dread. Ecstasy, eagerness, enjoyment, elation, enthrallment, enthusiasm, empathy, embarrassment, envy, enraged, euphoria, excitement, exhausted, exhilaration, exasperation. Fearful, fearlessness, friendliness, frustration, ferocity, fury, fondness. Gaiety, gleeful, glad, gloomy, gratitude, grief, guilt, grumpy. Happiness, hatred, helpless, hopeful, homesick, humiliated, horror, hostility, hurt, hysteria Indifference, interest, infatuation, isolation, irritation, insecurity, insult. Jealousy, joyful, jolly, joviality, jubilant. Love struck, liking, longing, loathing, lonely, loving, lustful Melancholy, mortified, mischievous and miserable. Neglected, nervous. Outraged, optimistic, overwhelmed. Panicky, passionate, powerless, pitiful, pleased, proud. Quiet, quick. Rage, regret, remorse, rapture, rejection, resentment, revulsion. Scorn, smug, suspicious, submissive, sentimental, sadness, satisfaction, shame, shock, shyness, sorrow, spite, sympathy, sexual desire, suspense, sentimental, stress, suffering, surprise. Tension, tenderness, torment, thrill, terror, triumphant, Vengeful. Uneasy, unhappy. Wonder, worry, woe, wrath. Zeal, zest.

\section{Observations}

Here are a few observations we make about the brain and nervous system.

Neurons are decision makers [2]. They decide to fire or not to fire. In the context of decision making, the study of neural firing belongs to the field of real rather than artificial intelligence.

The structure of the brain is stratified from very large neurons to minute ones with variable frequencies of firings. Because neurons and their firings are common to many animals and some animals' nervous systems can be so simple with few neurons, it is safe to assume that neural firing is mathematically the same and must itself be simple and very basic.

Eigenvalue theory [3] applies to the firing of neurons to derive Eigen functions as solutions of Fredholm's equation of the second kind and the inconsistency of numerical judgments does not play a significant role in the continuous case as it did in the discrete case of decision making.

The Eigen function solution [4] is a necessary condition for the existence of solutions to the response functional equation $w(a s)=b w(s)$ that characterizes the solvability of Fredholm's equation.

Linear combinations of such solutions such as,

$$
\begin{aligned}
& x_{1}, x_{2} \ldots, x_{p}, f=\alpha_{1} x_{1}+\alpha_{2} x_{2}+\ldots+\alpha_{p} x_{p} \\
& \|f\|_{k p}=\sum_{i=0}^{k}\left\|f^{(i)}\right\|_{p}=\sum_{i=0}^{k}\left(\int\left|f^{(i)}(t)\right|^{p} d t\right)^{1 / p)}
\end{aligned}
$$

are dense in Sobolev spaces that are among the most general kind of spaces used in mathematics to represent natural phenomena. Thus many of the functions encountered by the brain in the real world can be approximated arbitrarily close by such linear combinations. Linear combinations are fundamental in our mathematical thinking. Polynomials are linear combinations of powers of a variable. Polynomials are known to be dense in the space of continuous functions in the sense that they can be used to come as closely as desired to any function that has the property of continuity everywhere.

Fourier transforms give the space-time representation of the solutions and of a dense firings field that remain dense after transformation.

$$
F(w)=F[f(t)]=\int_{-\infty}^{+\infty} f(t) e^{-i w t} d t
$$

and its inverse

$$
f(t)=F^{-1}\left(f(w)=\frac{1}{2 \pi} \int_{-\infty}^{+\infty} F(\mathrm{w}) e^{i w t} d t\right.
$$

Because of the reciprocal condition that requires division, solutions are developed in the four division algebras: the real domain to represent the natural world, the complex domain to represent neural firings and their syntheses and feelings and imagination which are multiple valued, the quaternion domain to represent non-commutativity of modes of thought and action and the octonion domain to represent nonassociativity of modes of thought and action [5].

Solutions, which are functions of time, are indicated by making their two parameters functions of time.

How do firings and storage of information lead to remembering, sensing, feeling and thinking?

There is a need to create robots with finely responsive nonmechanistic behavior.

\section{Axioms for Functions of the Brain}

Despite its diversified functions and because of feedback the brain communicates with itself and arrives at a final overall feeling of a degree of dynamic satisfaction or dissatisfaction that connects with previous feeling. The brain uses the same electrical language for internal communications and interaction. The brain must operate with a simple common principle that allows it to both create the firings of neurons and to synthesize their signals meaningfully. The diversity of responses the brain makes are like playing the same composition of music on different instruments in an orchestra. Quality (timbre) makes different kinds of sound. Similarly, neural firings with different neurons and neurotransmitters create different kinds of feelings and imaginings. In the end all feelings and sensations thoughts and emotions arise from neural firings and how they are processed at synapses and are suspended there momentarily in the form of electromagnetic vibrations and the totality of these vibrations is again combined to obtain the diversity of activities of the brain. Each neuron has its characteristic firing function which if with changes would still have another characteristic function and so on. In other words, at any moment a neuron contributes its characteristic firing function and the brain must synthesize 
all the firing functions into a meaningful whole. We may deal with multifunction operators but not with multivariable functions.

At its initial internal communication at the moment of birth or before, when the brain becomes active with neural firings, the brain must communicate with itself meaningfully. Having no prior knowledge and experience it must compare and distinguish between signals. There is a field obtained from sensing, perceiving and feeling and internal organ functions at the subconscious level and conceptualizing, thinking, dreaming and muscular movement to fulfill intentions, needs and desires.

The brain is a nonlinear network with feedback. It cycles electricity and the outcome of diverse infinite cycling is an electric signal that is measured by amplitude and phase. Such a numerical outcome cannot be put into words in an explicit and clear way. Thus, in general, language and logic which are linear and of necessity proceed from "if" as an assumption to "therefore" as a conclusion cannot capture with sufficient accuracy and understanding, quantitative feedback signals. Unless we discover a lay language to describe magnitudes and phases in a way that we can all "understand", we would only be able to describe and understand (if that is ever possible) how the brain works in a technical mathematical way.

Long before measurement scales were invented, people had no direct way to perform measurement because they had no standards and had to compare things with each other to determine their relative order as described in Chapter 3 . We all have the ability to compare especially when we cannot measure things because we do not have the instrument or scale to do it. Another reason is that we may believe that the outcome of comparisons using our judgment would be calibrated better to our values than using a scale of measurement that was not devised for the particular use we are putting it to. A third reason may be that there is no known way to measure something like political effectiveness, happiness, aesthetic appeal and many other things.

Ancient people used their judgment to order things. The way they did it was to compare two things at a time to determine which was the larger or more preferred. By repeating the process they obtained a total ordering of the objects without assigning them numerical values. After being ordered they could rank them: first, second, and so on. But when many criteria are involved it is not so easy to combine the orders obtained with respect to each criterion to obtain a total order unless there is associated with each partial order a set of numbers that are in some sense commensurate so they can be combined using the numbers (weights or priorities) associated with the criteria.

\section{Conclusion}

We have provided a sketch for how to look at the nervous system and its byproducts of mind and feelings and how they function in a general philosophical way as a system with consciousness and with purpose. We also mentioned a diversity of situations and challenges of performance. Our goal in doing it was to aim at generality so the reader can learn to combine the more technical mathematical study of the nervous system with its major functions and purposes that make it amenable to a variety of interpretations. According to Waldman [6] consciousness exists at eight inclusive levels: reality (which Plato called Phenomena) consisting of the three space physical dimensions and time, and seven [7] states of consciousness (called by Plato Nuomena) that cover instinctual awareness (wakefulness), habitual responsiveness, intentional decision-making, free-floating imagination, self-reflective awareness, transformational awareness and enlightenment. Waldman's model of human consciousness consolidates more than 31,000 studies contained in the database of the National Library of Medicine. The first four levels seem to correspond to the perceptual-cognitive-active loop mentioned by Goertzel [1]:

"The quaternion structure is interpreted as a "perceptualcognitive-active loop," representing the basic structure of engagement with the world. This structure is seen to lead naturally to a certain type of adaptive learning, analogous to backtracking in artificial intelligence."

\section{References}

1. Goertzel B. On the algebraic structure of consciousness. Dynamical Psychology. 1996.

2. Saaty TL. Neurons the decision makers, Part I: The firing function of a single neuron. Neural Networks. 2017;86:102-14.

3. Saaty TL. The Neural Network Process (NNP): Generalization of the AHP and ANP to the continuous case of neural firing. RWS Publications. 2015.

4. Saaty TL. On the measurement of intangibles: A principal eigenvector approach to relative measurement derived from paired comparisons. Not Am Math Soc. 2013;60(2):192-208.

5. Saaty TL. Neurons the decision makers, Part II: The firings of many neurons and their density; the neural network its connections and field of firings. Neural Networks 2017;86:115-122.

6. Waldman MR. NeuroWisdom 101. E-book. 2014.

7. Saaty TL. Seven is the magic number in nature. Proc Am Philos Soc. 2016;160(4).

\section{*Correspondence to:}

Thomas Saaty

Distinguished University Professor

Fellow of the Center of the Philosophy of Science University of Pittsburgh

USA

Tel: 0014126216546

E-mail: saaty@katz.pitt.edu 